\title{
Association between Adiponectin and Low-Grade Albuminuria Is BMI-Dependent in Type 2 Diabetes
}

\author{
Drazenka Pongrac Barlovic Jelka Zaletel Janez Prezelj \\ Department of Endocrinology, Diabetes and Metabolic Diseases, University Medical Centre, Ljubljana, \\ Ljubljana, Slovenia
}

\section{Key Words}

Albuminuria - Adiponectin • Diabetes • Obesity · Insulin resistance

\begin{abstract}
Aim: Low-grade albuminuria is a marker of increased risk for both cardiovascular and renal disease. Adiponectin, with its insulin-sensitizing, anti-inflammatory and antiatherogenic properties, is associated with cardiovascular as well as renal disease. Limited and conflicting data exist on the association of adiponectin with low-grade albuminuria. Our aim was to explore the association of plasma adiponectin and lowgrade albuminuria in patients with type 2 diabetes. Furthermore, we were interested whether this association is dependent upon insulin sensitivity. Methods: In this cross-sectional study, plasma adiponectin and urinary albumin excretion rate (UAER) were determined in 71 patients by radioimmunoassay. Insulin sensitivity was measured by hyperinsulinemic euglycemic clamp and expressed as the $M$ value. The relationship between variables was described using univariate and multiple linear regression. Results: Adiponectin and UAER were negatively associated $(R=-0.285, p<0.05)$ only in patients with $\mathrm{BMI}>25$. The association was independent of the clamp-derived $\mathrm{M}$ value, gender, BMI, arterial pressure or cholesterol. Conclusion: In obese patients with type 2
\end{abstract}

diabetes, there is an inverse association between adiponectin and low-grade albuminuria, the association being independent of insulin resistance. The consequences of such a relationship in terms of renal disease progression and cardiovascular survival remain to be evaluated.

Copyright $\odot 2010$ S. Karger AG, Basel

\section{Introduction}

Plasma adiponectin is decreased in metabolic disorders, such as obesity, metabolic syndrome and diabetes [1]. Besides, adiponectin has insulin-sensitizing [2], antiatherogenic [3] and anti-inflammatory [4] properties. These features make adiponectin a promising candidate for explaining the link between the metabolic milieu and vascular disease. Indeed, in clinical studies, low plasma adiponectin was associated with increased cardiovascular risk [5, 6], but not always [7].

One of the established markers of increased cardiovascular risk is albuminuria [8]. Recently, it was shown that not only microalbuminuria, but also urinary albumin excretion rate (UAER) in the normal range imposes increased cardiovascular risk $[9,10]$.

Existing data on the association of circulating adiponectin with albuminuria are scarce. Clinical research was

\section{KARGER \\ Fax +41613061234 E-Mail karger@karger.ch} www.karger.com
(C) 2010 S. Karger AG, Basel

$1420-4096 / 10 / 0335-0405 \$ 26.00 / 0$

Accessible online at:

www.karger.com/kbr
Drazenka Pongrac Barlovic

Department of Endocrinology, Diabetes and Metabolic Diseases

University Medical Centre Ljubljana

Zaloska 7, SI-1000 Ljubljana (Slovenia)

Tel. +386 3134 8153, Fax +386 1522 2738, E-Mail drazenka.pongrac@ gmail.com 
primarily focused on high levels of UAER. Circulating adiponectin was found to be increased in patients with nephrotic range proteinuria as well as in patients with macroalbuminuria [11]. It was speculated that in these patients, characterized also by an increased cardiovascular risk, the increase in plasma adiponectin was compensatory and had vasculoprotective effects. On the other hand, the relationship between low levels of UAER and circulating adiponectin is less clear. Both an inverse relationship [12,13] and no relationship [14] between the two parameters have been reported. The relationship between adiponectin and low-grade albuminuria has attracted considerable interest since a recent study suggested that adiponectin may regulate podocyte function in mice [15]. Understanding the link between adiponectin and lowgrade albuminuria is important because new treatment options targeted at adiponectin function in low-grade albuminuria may prove beneficial, especially in patients with a high cardiovascular risk.

The purpose of our study was to assess the association between circulating adiponectin and UAER in low-albuminuric patients with increased cardiovascular risk, characterized by the presence of long-standing type 2 diabetes. Since both adiponectin [2] and UAER [16] have been linked to insulin resistance, we also aimed to analyze whether a possible association between plasma adiponectin and UAER, if present in these patients, is independent of insulin resistance.

\section{Patients, Materials and Methods}

We enrolled a cohort of 71 patients recruited in Slovenia for the DEMAND trial, a multicenter, randomized, prospective, double-blind study, conducted to examine the effect of delapril, an ACE inhibitor, used alone or in combination with manidipine, a calcium channel blocker, on GFR decline in hypertensive type 2 diabetic patients. The patients were 40 years of age or older, they had type 2 diabetes and arterial hypertension. Their serum creatinine was $<1.5 \mathrm{mg} / \mathrm{dl}(133 \mu \mathrm{mol} / \mathrm{l})$, and their UAER was in the normo- or microalbuminuric range in at least two of three consecutive overnight urine samples. Patients with non-diabetic renal disease, or some other serious or terminal concomitant disease, were not included. All subjects provided written informed consent. The study protocol was in accordance with the Declaration of Helsinki and was approved by the Slovenian Ethics Committee.

At the randomization visit, the subjects underwent physical examination, including measurements of height and weight. 24hour ambulatory blood pressure was measured by a portable automatic blood pressure monitor (SpaceLabs Healthcare, Issaquah, Wash., USA). A morning urine sample was first analyzed semiquantitatively (Multistix 10SG; Bayer, Germany). Patients with no findings suggestive of infection or non-diabetic glomerular dis- ease submitted three consecutive timed overnight urine samples on two occasions 2 months apart. UAER was measured by radioimmunoassay, and average UAER was calculated. Fasting venous blood was sampled from an antecubital vein for the measurement of HbAlc, creatinine and cholesterol. Total fasting plasma adiponectin was measured using a commercially available radioimmunoassay kit (Linco Research, St. Louis, Mo., USA). The inter- and intra-assay coefficients of variation were 1.8 and $9.25 \%$, respectively.

The patients discontinued treatment with ACE inhibitors, AT1-receptor antagonists and calcium receptor blockers at least 3 months before the randomization visit. At the time of randomization, they were under treatment for arterial hypertension with one or more antihypertensive drugs: 64 patients were receiving indapamide, 20 patients $\beta$-blockers, 22 patients $\alpha$-receptor antagonists, and 32 patients moxonidine (many of them were receiving a combination of these drugs). For the treatment of diabetes, the patients were receiving oral glucose-lowering agents and/or insulin: 37 patients were treated with insulin, 36 with metformin, 14 with repaglinide, 23 with sulfonylurea, and 5 with an $\alpha$-glucosidase inhibitor (many were receiving a combination of these drugs).

Insulin resistance was measured in the morning after a 12hour overnight fast [14] by the method of 2-hour hyperinsulinemic euglycemic clamp as described by DeFronzo et al. [17]. In short, a vein in the forearm was cannulated for infusion of insulin and glucose. Insulin (Actrapid; NovoNordisk, Denmark) was added to $50 \mathrm{ml}$ of saline solution in such a quantity that at an infusion a rate of $12.6 \mathrm{ml} / \mathrm{h}$ during the clamp procedure each patient received $2 \mathrm{mIU}$ of insulin per $1 \mathrm{~kg}$ of body weight per minute. At first, insulin was infused at a rate of $25.2 \mathrm{ml} / \mathrm{h}(4 \mathrm{mIU} / \mathrm{kg} / \mathrm{min})$ in order to suppress hepatic glucose output. After $10 \mathrm{~min}$, the infusion rate was lowered to $12.6 \mathrm{ml} / \mathrm{h}$ and then remained unchanged until the end of the procedure. Blood for the monitoring of glucose concentration was withdrawn at 5 -min intervals via a catheter in the contralateral antecubital vein. When blood glucose first reached a concentration of $95 \mathrm{mg} / \mathrm{dl}$, the start of the clamp was designated. For the next $120 \mathrm{~min}$, blood glucose was maintained in the range of euglycemia $(85-95 \mathrm{mg} / \mathrm{dl})$ by adjusting the rate of infusion of $20 \%$ glucose solution. The mean rate of glucose infusion during the last $30 \mathrm{~min}$ of the clamp procedure was expressed as the $\mathrm{M}$ value and used as the index of insulin sensitivity.

\section{Statistical Analysis}

All numerical variables were analyzed for the type of probability distribution using the Kolmogorov-Smirnov test. In normally distributed variables, the results were reported as mean \pm SD. Non-normally distributed variables were logarithmically transformed and reported as median (25th percentile, 75 th percentile). Univariate linear relationships between normally distributed variables were evaluated by Pearson's correlation coefficient. Univariate linear relationships between non-normally distributed variables were evaluated by Spearman's correlation coefficient. Multiple linear regression analysis was performed to evaluate the independent relationship of the studied variables. Statistical significance was considered at a $p$ value $<0.05$. All analyses were made using the Statistical Package for Social Sciences Version 11.0 (SPSS, Chicago, Ill., USA). 
Table 1. Demographic, clinical and biochemical characteristics of the study population

$\begin{array}{lc}\text { Patients (female/male) } & 71(36 / 35) \\ \text { Age, years } & 61 \pm 9 \\ \text { Diabetes duration, years } & 14 \pm 9 \\ \text { BMI, kg/m } & 30.5 \pm 5.1 \\ \text { 24-hour systolic BP, mm Hg } & 134 \pm 12 \\ \text { 24-hour diastolic BP, mm Hg } & 77 \pm 9 \\ \text { UAER, } \mu \mathrm{g} / \mathrm{min} & 4.0(2.7,12.4)^{1} \\ \text { Adiponectin, } \mu \mathrm{g} / \mathrm{ml} & 11.7 \pm 5.9 \\ \text { Glycated hemoglobin, \% } & 8.3 \pm 1.3 \\ \text { Total cholesterol, mmol/l } & 4.9 \pm 1.0 \\ \text { LDL cholesterol, mmol/l } & 2.8 \pm 0.8 \\ \text { HDL cholesterol, mmol/l } & 1.1(1.0,1.3)^{1} \\ \text { Triglycerides, } \mathrm{mmol} / \mathrm{l} & 1.5(1.1,2.2)^{1} \\ \text { Creatinine, } \mu \mathrm{mol} / \mathrm{l} & 76 \pm 14 \\ \text { M value, } \mathrm{mg} / \mathrm{kg} / \mathrm{min} & 5.5 \pm 3.1\end{array}$

${ }^{1}$ Data expressed as median (24th percentile, 75 th percentile).

Table 2. Multivariate analysis with adiponectin as a dependent variable in all patients

\begin{tabular}{llrr}
\hline $\begin{array}{l}\text { Dependent } \\
\text { variable }\end{array}$ & Independent variables & $\beta$ & $\mathrm{p}$ \\
\hline Adiponectin & UAER & -0.164 & 0.087 \\
& Sex (female vs. male) & 0.233 & 0.038 \\
& BMI & 0.257 & 0.016 \\
& M value & 0.262 & 0.024 \\
& HDL cholesterol & 0.458 & $<0.001$ \\
& Diastolic BP & -0.171 & 0.074 \\
\hline
\end{tabular}

$\mathrm{R}^{2}$ adjusted for the model $0.530(\mathrm{p}<0.001)$.

Table 3. Multivariate analysis with adiponectin as a dependent variable in patients with $\mathrm{BMI} \geq 25 \mathrm{~kg} / \mathrm{m}^{2}(\mathrm{n}=63)$

\begin{tabular}{llrr}
\hline $\begin{array}{l}\text { Dependent } \\
\text { variable }\end{array}$ & Independent variables & $\beta$ & $\mathrm{p}$ \\
\hline Adiponectin & UAER & -0.296 & 0.001 \\
& Sex (female vs. male) & 0.243 & 0.021 \\
& BMI & 0.223 & 0.020 \\
& M value & 0.134 & 0.166 \\
& HDL cholesterol & 0.507 & $<0.001$ \\
& Diastolic BP & -0.139 & 0.111 \\
\hline
\end{tabular}

$\mathrm{R}^{2}$ adjusted for the model $0.642(\mathrm{p}<0.001)$.

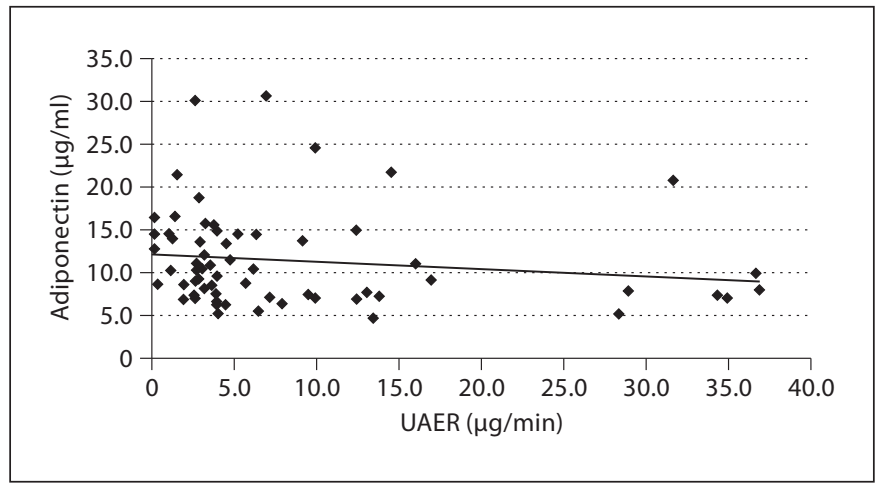

Fig. 1. Association between adiponectin and UAER in patients with $\mathrm{BMI}>25 \mathrm{~kg} / \mathrm{m}^{2}(\mathrm{R}=-0.285, \mathrm{p}=0.030)$.

\section{Results}

Basic demographic, clinical and biochemical characteristics of the patients are presented in table 1 . The patients studied had a median UAER of $4.0 \mu \mathrm{g} / \mathrm{min}$.

In the univariate linear regression analysis, adiponectin was associated with diastolic blood pressure $(\mathrm{R}=$ $-0.254, \mathrm{p}=0.046)$, HDL cholesterol $(\mathrm{R}=0.633, \mathrm{p}<0.001)$, and triglycerides $(\mathrm{R}=-0.307, \mathrm{p}=0.015)$, and was higher in women $(14.0 \pm 6.2 \mu \mathrm{g} / \mathrm{ml})$ than in men $(9.4 \pm 4.6 \mu \mathrm{g} /$ $\mathrm{ml}, \mathrm{p}=0.001)$. Adiponectin was not associated with UAER, the clamp-derived $M$ value ( $\mathrm{p}=\mathrm{NS}$ ), or with BMI. $\mathrm{BMI}$ and the clamp-derived $\mathrm{M}$ value were negatively associated $(R=-0.332, p=0.005)$.

In the multiple linear regression model, adiponectin was entered as a dependent variable (table 2). Gender, BMI, diastolic blood pressure, HDL cholesterol and GFR were included as independent variables, known to be associated with plasma adiponectin. UAER and $M$ values were added in the model because of our interest in the association of low-grade albuminuria and insulin resistance with adiponectin. UAER was not significantly associated with adiponectin. However, $M$ value, gender, BMI and HDL cholesterol were significant predictors of adiponectin, explaining more than $50 \%$ of its variability.

Most patients in our study were overweight or obese (BMI $>25, n=63$ ). Because of the strong association of adiponectin with BMI and BMI with albuminuria, to account for BMI, we decided to analyze the relationship between adiponectin and UAER separately in subjects with BMI $>25$. When patients with BMI $>25$ were analyzed separately $(n=63)$, there was a statistically significant negative association between adiponectin and UAER $(\mathrm{R}=-0.285, \mathrm{p}<0.05)$ in the univariate analysis (fig. 1). In 
the multiple linear regression model, the negative association between adiponectin and UAE was statistically significantly independent of gender, BMI, blood pressure, HDL cholesterol, GFR and M value (table 3). In this group of patients, adiponectin was not associated with the $M$ value.

In patients with BMI $<25(\mathrm{n}=8)$, there was no association between adiponectin and low-grade albuminuria. These patients did not differ significantly from those with BMI $>25$ in any of the examined clinical and laboratory parameters.

\section{Discussion}

The results of our study show that there is a negative association between plasma adiponectin and UAE in overweight and obese patients with type 2 diabetes and low-grade albuminuria. This association was independent of insulin resistance and other factors known to affect plasma adiponectin, such as gender, BMI, blood pressure and HDL cholesterol.

Adiponectin is one of the most interesting adipocytokines, mostly due to its beneficial and protective vascular effects. In one study, adiponectin knockout mice showed twice as much neointimal formation in response to external vascular injury as wild-type mice [18]. Neointimal proliferation in adiponectin knockout mice was attenuated by adenovirus-mediated adiponectin administration [19]. Also, globular adiponectin was found to protect apolipoprotein E-deficient mice from atherosclerosis [3]. The vasculoprotective properties of adiponectin have been confirmed in clinical studies as well. In a prospective study, men with high plasma adiponectin were at lower risk of myocardial infarction than those with medium or low plasma adiponectin levels [20]. The association between high plasma adiponectin and a low incidence of cardiovascular disease was found also in other patient populations, including those with renal disease [6]. However, high plasma adiponectin was not consistently shown to act as a vasculoprotective substance. In some studies in patients undergoing coronary angiography [7] and in patients with chronic kidney disease [21], high adiponectin levels were associated with increased cardiovascular risk. These data suggest that simply plasma concentration of this adipocytokine may not always directly reflect its mechanisms of action.

On the other hand, increased albuminuria is a wellrecognized early marker of cardiovascular disease [22]. A positive relationship between plasma adiponectin and albuminuria has been demonstrated in patients with nephrotic range proteinuria [10] and also in patients with proteinuria well below the nephrotic range (approx. $1 \mathrm{~g} /$ day) [23]. It has been suggested that in kidney disease, adiponectin may improve endothelial function [24] that is impaired in advanced stages of disease. In line with these clinical findings, supporting an active protective role of adiponectin in kidney disease, are the results of studies in adiponectin-knockout mice. These animals were found to have increased albuminuria and tubulointerstitial fibrosis compared to wild-type mice. In addition, treatment of adiponectin knockout mice with adenovirus-mediated adiponectin resulted in a decrease in albuminuria as well as in a decrease in markers of inflammation and oxidative stress $[25,26]$. Another study, performed on cultured podocytes by Sharma et al. [15], also showed that adiponectin acts directly on the renal podocyte cell and reduces its permeability to albumin through reduction of oxidative stress.

In contrast to investigations in patients with high UAER, studies examining the association of plasma adiponectin with low-grade UAER yielded conflicting results. In hypertensive patients with microalbuminuria, plasma adiponectin was lower compared to hypertensive patients with normoalbuminuria or to healthy controls [12]. Similarly, an inverse relationship of plasma adiponectin with low-grade UAER was present in AfricanAmerican obese patients [15]. On the other hand, in type 2 diabetic patients with normo- or microalbuminuria [14], no association was found between plasma adiponectin and UAER. However, the last two studies were not designed primarily to evaluate the relationship between adiponectin and albuminuria, which may be one of the reasons that no associations were found.

In our study, also examining patients with type 2 diabetes and low-grade albuminuria, there was no association between adiponectin and UAER. However, when only overweight and obese patients were included in the analysis, there was a negative association between adiponectin and UAER. This association was independent of other factors reported to influence the relationship between adiponectin and albuminuria, such as BMI, blood pressure and cholesterol. Similarly, in patients without diabetes, a negative relationship between adiponectin and albuminuria was demonstrated only in obese patients [27]. The negative association of adiponectin and low-grade albuminuria may have some speculative explanations. Most likely, in our patients with diabetes, arterial hypertension, dyslipidemia and obesity, the process of atherosclerosis was expressed at the systemic endothe- 
lial level. Adiponectin may accumulate in the vascular subendothelial space, where the endothelial barrier is damaged [28], as has been shown for patients with coronary heart disease [29]. In this way, adiponectin could be lost in subendothelial space, and its level in plasma could correlate with the degree of vascular damage, as marked by albuminuria.

According to the results of our study and the published data, the relationship of plasma adiponectin with albuminuria appears to change in the course of the development of kidney damage. At first, plasma adiponectin decreases with increasing albuminuria, but this relationship is later reversed. According to the results of our study and the published data, we could speculate that adiponectin becomes positively associated with albuminuria in the course of the development of macroalbuminuria. This described relationship of albuminuria with adiponectin could even support the hypothesis that the development of macroalbuminuria through microalbuminuria is not simply a linear process, and that different pathophysiological mechanisms may underlie each of the stages in the development of kidney disease in type 2 diabetes. Further prospective studies are needed to establish and confirm this threshold point and to better understand the role of adiponectin in micro- and macrovascular diseases.

Since adiponectin is associated with both albuminuria and insulin resistance, and albuminuria is closely linked to insulin resistance, we speculated that the mechanism linking adiponectin and albuminuria could be insulin resistance. However, when a clamp-derived $M$ value was entered in the multiple linear regression model together with UAER, the association of plasma adiponectin with low-grade albuminuria was independent of insulin resistance. This is in line with a study in healthy individuals where the association of plasma adiponectin with endothelium-dependent vasodilatation was independent of insulin sensitivity [30].

We found no significant association between adiponectin and $\mathrm{BMI}$ in the univariate regression analysis in the patients studied. It is possible that there was no significant association of adiponectin with BMI because adiponectin is associated with insulin sensitivity and insulin sensitivity is associated with BMI. Although it is well accepted that plasma adiponectin levels are decreased in obesity [1], our results show a positive association between adiponectin levels and BMI. We think this is because our patients were mostly obese with an average $\mathrm{BMI}$ of 30.5 , and that in this mostly obese population the association between adiponectin and BMI is not the same as if we studied subjects with a wide range of BMI. Also, we cannot exclude the possibility that measuring different adiponectin fractions in our patient population, for example high molecular weight adiponectin could result in different associations with BMI.

Although the methods used in our study were carefully selected and performed with utmost care, some limitations of the study should be acknowledged. First, the size of the study population was relatively small, and thus our conclusions should be validated in larger scale studies. Also, due to the cross-sectional design, no conclusions regarding the cause-and-effect relationship can be drawn from this study. Longitudinal studies with evaluation of plasma adiponectin changes accompanying advancing kidney disease are needed. Furthermore, prospective studies with the evaluation of cardiovascular status could explain the role of adiponectin and low-grade albuminuria in terms of cardiovascular outcomes. Another possible limitation could be that the assay used in this study measures total adiponectin and not individual adiponectin fractions. The relationships described could be different if only high molecular weight adiponectin were investigated. However, most other studies investigating the level of adiponectin in renal disease have used total adiponectin assay, which is important for comparisons of the results to be possible.

In conclusion, we found a negative association of adiponectin with low-grade albuminuria in overweight and obese patients with type 2 diabetes, but not in patients with normal weight. This association was independent of the link of adiponectin with insulin sensitivity. Further prospective studies are needed to better clarify the causative role of adiponectin in albuminuria in obesity and the possible clinical relevance of this association.

\section{Acknowledgements}

The authors are grateful to Giuseppe Remuzzi and Piero Ruggenenti who organized the DEMAND study.

References

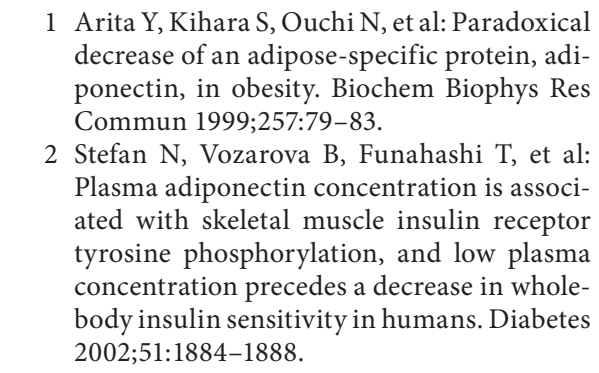

Kidney Blood Press Res 2010;33:405-410 409 
-3 Yamauchi T, Kamon J, Waki H, et al: Globular adiponectin protected ob/ob mice from diabetes and apo-E-deficient mice from atherosclerosis. J Biol Chem 2003;278:24612468.

4 Kumada M, Kihara S, Ouchi N, et al: Adiponectin specifically increased tissue inhibitor of metalloproteinase-1 through interleukin-10 expression in human macrophages. Circulation 2004;109:2046-2049.

$\checkmark 5$ Kumada M, Kihara S, Sumitsuji S, et al: Association of hypoadiponectinaemia with coronary artery disease in men. Arterioscler Thromb Vasc Biol 2003;23:85-89.

6 Zoccali C, Mallamaci F, Tripepi G, et al: Adiponectin, metabolic risk factors, and cardiovascular events among patients with endstage renal disease. J Am Soc Nephrol 2002; 13:134-141.

7 Pilz S, Mangge H, Wellnitz B, et al: Adiponectin and mortality in patients undergoing coronary angiography. J Clin Endocrinol Metab 2006;91:4277-4286.

$\checkmark 8$ De Zeueuw D, Parving HH, Henning RH, et al: Microalbuminuria as an early marker for cardiovascular disease. J Am Soc Nephrol 2006;17:2100-2105.

-9 Wachtell K, Wachtell K, Ibsen H, et al: Albuminuria and cardiovascular risk in hypertensive patients with left ventricular hypertrophy: the LIFE study. Ann Intern Med 2003;139:901-906.

-10 Zoccali C, Mallamaci F, Panuccio V, et al: Adiponectin is markedly increased in patients with nephrotic syndrome and is related to metabolic risk factors. Kidney Int 2003; 63(suppl 84):98-102.

-11 Looker H, Krakoff J, Funahashi T, et al: Adiponectin concentrations are influenced by renal function and diabetes duration in Pima Indians with type 2 diabetes. J Clin Endocrinol Metab 2004;89:4010-4017.
12 Tsioufis C, Dimitriadis K, Chatzis D, et al: Relation of microalbuminuria to adiponectin and augmented C-reactive protein levels in men with essential hypertension. Am J Cardiol 2005;96:946-951.

13 Barlovic DP, Zaletel J, Prezelj J: Adipocytokines are associated with renal function in patients with normal range glomerular filtration range and type 2 diabetes. Cytokine 2009;46:142-145.

14 Hotta K, Funahashi T, Arita Y, et al: Plasma concentrations of a novel, adipose-specific protein, adiponectin, in type 2 diabetic patients. Arterioscler Thromb Vasc Biol 2000; 20:1595-1599.

15 Sharma K, Ramachandrarao S, Qiu G, et al: Adiponectin regulates albuminuria and podocyte function in mice. J Clin Investig 2008;118:1645-1656.

16 DeCosmo S, Minenna A, Ludovico O, et al: Increased urinary albumin excretion, insulin resistance, and related cardiovascular risk factors in patients with type 2 diabetes Diabetes Care 2005;28:910-915.

17 DeFronzo RA, Tobin JD, Andres R: Glucose clamp technique: a method for quantifying insulin secretion and resistance. Am J Physiol 1979;237:214-223.

18 Kubota N, Tearuchi Y, Yamauchi T, et al: Disruption of adiponectin causes insulin resistance and neointimal formation. J Biol Chem 2002;277:25863-25866.

19 Matsuda M, Shimomura I, Sata M, et al: Role of adiponectin in preventing vascular stenosis. The missing link of adipo-vascular axis. J Biol Chem 2002;277:37487-37491.

20 Pischon T, Girman CJ, Hotamisligil GS, et al: Plasma adiponectin levels and risk of myocardial infarction in men. JAMA 2004;291: 1730-1737.

-21 Menon V, Li L, Wang X, Greene T, et al: Adiponectin and mortality in patients with chronic kidney disease. J Am Soc Nephrol 2006;17:2599-2606.

-22 De Zeueuw D, Parving HH, Henning RH, et al: Microalbuminuria as an early marker for cardiovascular disease. J Am Soc Nephrol 2006;17:2100-2105.
23 Guebre-Egziabher F, Bernhard J, Funahashi $\mathrm{T}$, et al: Adiponectin in chronic kidney disease is related more to metabolic disturbances than to decline in renal function. Nephrol Dial Transplant 2005;20:129-134.

24 Malyszko J, Malyszko JS, Brzosko S, et al: Adiponectin is related to CD146, a novel marker of endothelial cell activation/injury in chronic renal failure and peritoneally dialyzed patients. J Clin Endocrinol Metab 2004;89:4620-4627.

25 Ohashi K, Iwatani H, Kihara S, Nakagawa Y, Komura N, Fujita K, et al: Exacerbation of albuminuria and renal fibrosis in subtotal renal ablation model of adiponectin-knockout mice. Arterioscler Thromb Vasc Biol 2007; 27:1910-1917.

26 Koshimura J, Fujita H, Narita T, et al: Urinary adiponectin excretion is increased in patients with overt diabetic nephropathy. Biochem Biophys Res Commun 2004;316: 165-169.

$>27$ Yano Y, Hoshide S, Ishikawa J, et al: Differential impacts of adiponectin on low-grade albuminuria between obese and nonobese persons without diabetes. J Clin Hypertens (Greenwich) 2007;9:775-782.

28 Okamoto Y, Arita Y, Nishida M, et al: An adipocyte-derived plasma protein, adiponectin, adheres to injured vascular walls. Horm Metab Res 2000;32:47-50.

$\checkmark 29$ Kojima S, Funahashi T, Sakamoto T, et al: The variation of plasma concentrations of a novel, adipocyte derived protein, adiponectin, in patients with acute myocardial infarction. Heart 2003;89:667-668.

30 Fernandez-Real JM, Castro A, Vazquez G, et al: Adiponectin is associated with vascular function independent of insulin sensitivity. Diabetes Care 2004;27:739-745. 